\title{
The Val/Met Polymorphism of the Brain-Derived Neurotrophic Factor (BDNF) Gene Predicts Decline in Perceptual Speed in Older Adults
}

\author{
Paolo Ghisletta \\ University of Geneva and Distance Learning University \\ Lars Bertram \\ Max Planck Institute for Molecular Genetics, Berlin, Germany \\ Denis Gerstorf \\ Humboldt University and Pennsylvania State University
}

\author{
Lars Bäckman \\ Karolinska Institute, Stockholm, Sweden
}

Andreas Markus Brandmaier

Max Planck Institute for Human Development, Berlin, Germany

Tian Liu

Max Planck Institute for Human Development, Berlin, Germany, and Max Planck Institute for Molecular Genetics, Berlin,

Germany

\author{
Ulman Lindenberger \\ Max Planck Institute for Human Development, Berlin, Germany
}

\begin{abstract}
The brain-derived neurotrophic factor (BDNF) promotes activity-dependent synaptic plasticity, and contributes to learning and memory. We investigated whether a common Val66Met missense polymorphism (rs6265) of the BDNF gene is associated with individual differences in cognitive decline (marked by perceptual speed) in old age. A total of 376 participants of the Berlin Aging Study, with a mean age of 83.9 years at first occasion, were assessed longitudinally up to 11 times across more than 13 years on the Digit-Letter task. Met carriers $(n=123,34 \%)$ showed steeper linear decline than Val homozygotes $(n=239,66 \%)$; the corresponding contrast explained $2.20 \%$ of the variance in change in the entire sample, and $3.41 \%$ after excluding individuals at risk for dementia. These effects were not moderated by sex or socioeconomic status. Results are consistent with the hypothesis that normal aging magnifies the effects of common genetic variation on cognitive functioning.
\end{abstract}

Keywords: cognitive decline, BDNF Val/Met polymorphism, Berlin Aging Study

In recent years, the field of cognitive aging has enriched its explanatory scope by paying increasing attention to the effects of genetic and epigenetic variation on individual differences in cognitive performance. Two broad approaches can be distinguished
(Harris \& Deary, 2011; Lindenberger et al., 2008; Payton, 2009; Neiderhiser, 2001; Plomin, DeFries, Knopik, \& Neiderhiser, 2013; Plomin, Haworth, Meaburn, Price, Wellcome Trust Case Control Consortium 2, \& Davis, 2013; Turkheimer, 2011). On the one
This article was published Online First March 24, 2014.

Paolo Ghisletta, Faculty of Psychology and Educational Sciences, University of Geneva, Geneva, Switzerland, and Distance Learning University, Brig, Switzerland; Lars Bäckman, Aging Research Center, Karolinska Institute, Stockholm, Sweden; Lars Bertram, Neuropsychiatric Genetics Group, Max Planck Institute for Molecular Genetics, Berlin, Germany; Andreas Markus Brandmaier, Center for Lifespan Psychology, Max Planck Institute for Human Development, Berlin, Germany; Denis Gerstorf, Department of Psychology, Humboldt University, Berlin, Germany, and Department of Human Development and Family Studies, Pennsylvania State University; Tian Liu, Center for Lifespan Psychology, Max Planck Institute for Human Development and Neuropsychiatric Genetics Group, Max Planck Institute for Molecular Genetics; Ulman Lindenberger, Center for Lifespan Psychology, Max Planck Institute for Human Development.

This article reports data from the Berlin Aging Study (BASE; www .base-berlin.mpg.de). The BASE was initiated by the late Paul B. Baltes
(Psychology) in collaboration with Hanfried Helmchen (Psychiatry), Elisabeth Steinhagen-Thiessen (Internal Medicine and Geriatrics), and Karl Ulrich Mayer (Sociology). Financial support came from the Max Planck Society, the Free University of Berlin, the German Federal Ministry for Research and Technology (1989-1991, 13 TA 011 + 13 TA 011/A), the German Federal Ministry for Family, Senior Citizens, Women, and Youth (1992-1998, 314-1722-102/9 + 314-1722-102/9a), the BerlinBrandenburg Academy of Sciences' Research Group on Aging and Societal Development (1994-1999), and from the German Ministry for Education and Research (BMBF grant \#16SV5538 to Lars Bertram). The authors acknowledge additional support from the Max Planck Society and the German Research Foundation (Cooperative Research Grant SFB 378).

Correspondence concerning this article should be addressed to Paolo Ghisletta, Faculty of Psychology and Educational Sciences, University of Geneva, Boulevard du Pont d'Arve 40, 1211 Geneva, Switzerland. E-mail: paolo.ghisletta@unige.ch 
hand, behavior-genetic approaches rely on twin and adoption studies to estimate the influence of genetic and environmental effects on interindividual differences in given phenotypical traits of interest in a particular population. Molecular approaches, on the other hand, rely on molecular genetics to identify a more or less large number of genes, assessed through the collection of DNA, that regulate the expression of behavioral traits of interest. Although quantitative studies in humans, such as on the heritability of psychiatric disorders, appeared in the literature nearly 50 years ago (Heston, 1966), molecular studies are much younger. Nevertheless, the proliferation of molecular genetic studies in fields such as medicine and biology is spreading over to studies of cognition (Payton, 2009; Plomin, Haworth, et al., 2013). The two approaches have also been combined; for instance, Davies et al. (2011) used molecular approaches to estimate heritability in crystallized and fluid intelligence.

In molecular genetic research, two different strategies can be set apart. The first is exploratory in nature and consists of discovering associations between variability in a given trait and thousands of common DNA markers, most frequently single-nucleotide polymorphisms (SNPs). This method, called genome-wide association study (GWAS), has become quite popular because several technological advances have drastically reduced costs involved to collect genome data. It is estimated that in the past 5 years, over 200 such associations have been published (Plomin, Haworth, et al., 2013). The second method is more confirmatory in nature and consists of choosing a priori the gene(s) whose expression is hypothesized to be associated with variation in the trait under investigation. This candidate gene approach requires prior knowledge about the gene's biological influence on the trait or about the discrepant allelic expressions associated with variations in the trait.

One gene that has received much attention in the cognitive literature is the brain-derived neurotrophic factor (BDNF) gene, located on chromosome 11 in humans. The BDNF gene regulates the homonymous protein, to which multiple functions are attributed, most notably neuronal growth and differentiation, neuronal plasticity and survival, and oxidative stress (Payton, 2009). The Val66Met (rs6265) SNP, a common methionine (Met) substitution for valine (Val) at codon 66, is probably the most investigated SNP of this gene (Harris \& Deary, 2011; Payton, 2009). The first study to establish an association between the Val66Met BDNF polymorphism and human memory was published by Egan et al. (2003), who showed that Met allele carriers had lower delayed episodic memory performance than Val homozygotes. Since then, several studies have shown the Val66Met SNP of the BDNF gene to be associated with episodic memory (Dempster et al., 2005; Echeverria et al., 2005; Hariri et al., 2003; Li et al., 2010; Miyajima et al., 2008; Tan et al., 2005), delayed recall (Miyajima et al., 2008), working memory (Echeverria et al., 2005; Rybakowski, Borkowska, Czerski, Skibińska, \& Hauser, 2003; Rybakowski et al., 2006), general intelligence (Tsai, Hong, Yu, \& Chen, 2004), and perceptual speed (Miyajima et al., 2008; Raz, Rodrigue, Kennedy, \& Land, 2009). In most cases, the adenine allele (which codes for Met) is associated with reduced cognitive performance compared with the guanine allele (coding for Val). Moreover, it has been shown that Met carriers have smaller hippocampal volumes than Val homozygotes, independent of age and gender (Bueller et al., 2006; Frodl et al., 2007; Pezawas et al., 2004), altered hippocampal activation patterns (Lövdén et al., 2011), and reduced hippocampal neuronal integrity (Egan et al. (2003)). Lim et al. (2013) found that in healthy adults with high beta-amyloid, Met carriers showed poorer cognitive performance and greater hippocampal atrophy than Val homozygotes; in adults with low beta-amyloid, this genetic effect was not reliable. Consistent with these results is that the Met allele impairs activity-dependent secretion of BDNF (Egan et al. (2003)), which may impact on early and permanent changes in cellular development and plasticity (Miyajima et al., 2008).

Moreover, the effect of the BDNF gene on cognitive performance may be magnified in old age, in line with the resource modulation hypothesis of genetic effects on cognition put forward by Lindenberger et al. (2008). According to this hypothesis, the greater variability in observed cognitive performance in old, compared with younger, ages may partially originate from common genetic polymorphisms whose effect is magnified in old age because of reduced brain resources. This greater effect of polymorphisms in older, compared with younger, ages thus increases the between-person differences. Empirical evidence in support of this hypothesis is recent but consistent (e.g., Colzato, van den Wildenberg, \& Hommel, 2013; Li et al., 2013; Papenberg et al., 2013). For instance, Li et al. (2010) showed that whereas older-adult Met-carriers of the BDNF gene performed less well than olderadult Val homozygotes on a task of backward serial recall, in younger adults, this effect was not observed. Sambataro et al. (2010) showed, for a declarative memory task, that the negative age gradient in hippocampal activation was greater in BDNF rs6265 Met-carriers than in Val homozygotes. Thus, the effect of the Val66Met missense polymorphism in the BDNF gene on cognitive performance may be greater in older than in younger ages.

A major objective of cognitive aging research consists of understanding predictors and correlates of change in cognitive performance during adulthood and old age. To further our knowledge of genetic effects on cognitive decline, we desperately are in need of longitudinal studies that assess genetic information and repeated cognitive assessments. Nevertheless, as Payton (2009) pointed out, revealing genetic effects on cognitive decline requires large longitudinal studies lasting decades, with several testing sessions. In recent years, many large-scale longitudinal studies have become available (for reviews, see Hultsch, 2004 and Schaie \& Hofer, 2001). Few of these, however, have collected samples allowing for individual genome analyses. In particular, little work exists investigating associations between the Val66Met SNP of the BDNF gene and change in, rather than level of, cognitive performance. Erickson et al. (2008) examined the association between the BDNF gene and performance in executive function over a 10-year period. Met carriers had lower scores at the beginning of the study and did not decline, whereas Val homozygotes had higher scores at the beginning but then declined. However, the Erickson et al. (2008) study included a small sample $(N=53)$, which was measured only twice and was younger than the sample reported here.

\section{Objectives}

Using a candidate gene approach, we focused on the BDNF gene and its Val66Met SNP to investigate its association with change in cognitive performance in the Berlin Aging Study (BASE; Baltes \& Mayer, 1999; Lindenberger, Smith, Mayer, \& 
Baltes, 2010). Based on the resource modulation hypothesis, we predicted that Val homozygotes would decline less in cognitive performance than Met carriers. To study change in cognitive performance, we applied the linear mixed effects model (Laird \& Ware, 1982). Characteristics about change are estimated at the latent level, thereby isolating reliable from residual variance in the task, which maximizes the power to detect interindividual variability in change and, subsequently, effects of possible predictors of interindividual variability in change. The novel features of this study are a relatively large sample size, a high number of repeated measures, a relatively long epoch of observation, the statistical control of multiple potential age confounds, and the use of a statistical method that accounts for residual variance.

\section{Method}

\section{Participants}

The initial sample of the Berlin Aging Study (BASE) included 516 German (at that time, West German) White European participants and was stratified by age (ranging from 70 to 103 years) and sex (Baltes \& Mayer, 1999). There were six waves of measurements, each with an initial assessment (IA) and a broader intensive protocol (IP), except for the second, which was limited to the IA. Thus, there were 11 total repeated assessments. For an in-depth analysis of longitudinal sample attrition, which separates mortality-associated and experimental components, see Lindenberger, Singer, and Baltes (2002).

Genetic information about BDNF was available for 362 participants (for more details, see Genotyping section). Thus, our analyses are limited to this subsample, which represents $70 \%$ of the initial BASE sample. This sample had a mean initial age of 83.88 years $(S D=8.70)$. Table 1 includes the longitudinal sample size, the average age, and the average duration in the study since study inception for this subsample.

\section{Cognitive Tasks and Procedure}

The longitudinal battery of the BASE spans over six full occasions, all of which but one are composed of two subsessions. An in-depth description of the cognitive battery assessed in the BASE and its psychometric properties was provided in Lindenberger, Mayr, and Kliegl (1993). Here we focus on the Digit-Letter (DL) task, which was one of the three tasks to assess perceptual speed and the only task assessed at each subsession. This task is very similar to the Digit-Symbol Substitution Test of the Wechsler Adult Intelligence Scale (WAIS; Wechsler, 1955). Participants were shown digits and asked to name the letters (instead of symbols) associated with those digits, according to a template. The template was visible during the whole duration of the task, which consisted of 21 sheets, each presenting six items. Participants were assessed individually at their homes by trained testers. Participants in need wore prescription glasses and/or hearing aids. Visually impaired participants were read the items by the testers. All participants gave answers orally.

We focus on the DL task for three reasons: (a) its number of assessments, which is higher than all other tasks in the battery $(n=$ 11); (b) its psychometric properties (at Time 1, Cronbach's alpha $=.93$; intercoder reliability Pearson correlation $=1.00$; and

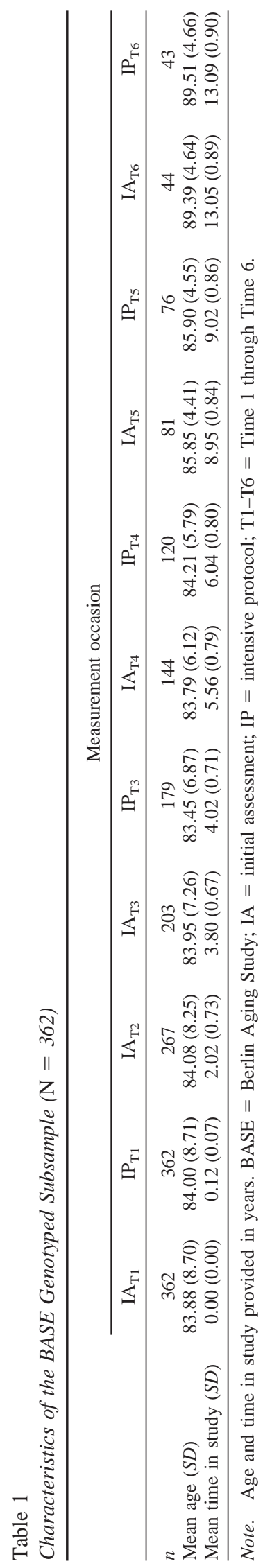


Stuart's rank correlation $=.99$; see Lindenberger et al., 1993), which are the best compared with all other cognitive tasks in the battery; and (c) its strong loading on a factor of general cognitive change (.96; whereas for other BASE tasks, the analogous standardized loadings averaged .72; see Lindenberger \& Ghisletta, 2009). Thus, the DL is the task that was most often assessed and best measured, and that provides the most reliable information about change in cognitive performance. For these three reasons, the DL test is arguably the best marker of cognitive aging in this sample. In addition, the results of a recent analysis of twin data suggest that genetic variance in perceptual speed drives age changes in spatial and memory abilities (Finkel, Reynolds, McArdle, Hamagami, \& Pedersen, 2009), in line with the processing speed theory of cognitive aging (Salthouse, 1996).

The scores analyzed here are the total number of correct responses after $3 \mathrm{~min}$. For ease of interpretation, the scores were T-standardized $(M=50, S D=10)$ with the first wave as anchor (to conserve changes in the mean and variance). Incomplete data were not imputed, and no participant was excluded from the analyses.

\section{Covariates}

To avoid confounding effects in the analyses, we controlled for sex and socioeconomic status (SES; see Raz et al., 2009, and Turkheimer, Haley, Waldron, D’Onofrio, \& Gottesman, 2003, respectively, for differential genetic effects on cognition across sexes or SES strata). The sample included 190 males and 172 females. The SES variable was a composite, summarizing number of years of formal education, social class, occupational prestige, and most recent income (compare Baltes \& Mayer, 1999, and Lindenberger et al., 2002). We also controlled for probable dementia diagnosis, an indicator introduced by Gerstorf, Herlitz, and Smith (2006), based on age cohort-specific cutoffs of the Short Mini Mental State Examination that were validated by independent clinical diagnoses of dementia performed by geriatricians (Helmchen et al., 1999). The subsample considered here counted 88, 71, 47, 27, 14, and 5 participants likely affected by dementia at IA Time 1 to Time 6, respectively. Overall, 127 (35\%) participants were likely affected by dementia at one or more measurement occasions.

To avoid underestimating the effects of aging, we also estimated retest effects with retest markers. At all occasions but the first, we included a dummy variable to indicate whether the task had been administered previously $(1=y e s, 0=n o)$. This technique is widely applied and has proven useful in a number of independent studies with age heterogeneous samples (e.g., Ferrer, Salthouse, McArdle, Stewart, \& Schwartz, 2005; Ghisletta, Rabbitt, Lunn, \& Lindenberger, 2012; Lindenberger \& Ghisletta, 2009; Lövdén, Ghisletta, \& Lindenberger, 2004; McArdle, Prescott, Hamagami, \& Horn, 1998). We also tested a single retest indicator counting the maximum number of previous exposures to the task. ${ }^{1}$ This second coding assumes retest effects to increase linearly with the number of previous exposures and allows estimating variability across participants in retest effects (which is not possible for the ensemble of former retest effects). We tested both occasionspecific and linearly increasing retest effects and found the former to be statistically more adequate.

\section{Genotyping}

The first IP of the BASE consisted of 14 sessions (to assess various psychological, sociological, psychiatric, internal medicine, and geriatric constructs). At Sessions 10 and 11, during the anamnesis and the physical examination, trained nurses drew $50 \mathrm{ml}$ of blood from participants. The SNP rs6265 (also known as Val66Met), located near the BDNF gene in chromosome 11, was genotyped using the Taqman 5 '-exonuclease allelic discrimination assay (see Holland, Abramson, Watson, \& Gelfand, 1991). The frequencies of the BDNF rs6265 genotypes were $66 \%(n=239)$ Val/Val (GG allele homozygotes), 31\% $(n=111) \mathrm{Val} / \mathrm{Met}$, and $3 \%(n=12)$ Met/Met (A allele homozygotes). These frequencies are in Hardy-Weinberg equilibrium. For analysis purposes, we collapsed heterozygotes and Met homozygotes into one group of Met (A allele) carriers $(34 \%, n=123)$, given the very low frequency of Met/Met homozygous participants.

\section{Data Analysis}

We used linear mixed effects models (LMEMs; Laird \& Ware, 1982) to study how cognitive performance evolved across the repeated measures. This type of model has become prevalent for the analyses of repeated-measures data and extends the ordinary least-squares regression model to accommodate data that are inherently dependent (due to the repeated assessments of each individual). For any chosen predictor, both fixed (sample averages) and random (individual deviations from sample averages) effects can be specified. If the latter result is significant, the model can be expanded to include additional predictors of such individual differences (Snijders \& Bosker, 1999). LMEMs have been shown to be more powerful than repeated-measures analysis of variance in detecting predictors of individual differences in change (Fan, 2003) and to better accommodate incomplete data (Snijders \& Bosker, 1999). LMEMs are also called hierarchical linear models because the data to which they are applied are often organized on multiple levels. Here, the repeated measures are said to constitute the Level 1 of the data hierarchy, whereas individuals are on Level 2 (Bryk \& Raudenbush, 1987).

We tested the model represented by the following equation:

$$
\begin{aligned}
Y_{i j}= & I_{j}+L S_{j} \cdot \bar{a}_{i j}+Q S_{j} \cdot \bar{a}_{i j}^{2}+\beta_{(1, \ldots, 10)} \cdot r_{(1, \ldots, 10) j} \\
& +\beta_{11} \cdot d d_{j}+\beta_{12} \cdot \operatorname{sex}_{j}+\beta_{13} \cdot S E S_{j} \\
& +\beta_{14} \cdot d d_{j} \cdot \bar{a}_{i j}+\beta_{15} \cdot \operatorname{sex}_{j} \cdot \bar{a}_{i j}+\beta_{16} \cdot S E S_{j} \cdot \bar{a}_{i j} \\
& +\beta_{17} \cdot d d_{j} \cdot \operatorname{sex}_{j}+\beta_{18} \cdot d d_{j} \cdot S E S_{j}+\beta_{19} \cdot \operatorname{sex}_{j} \cdot S E S_{j} \\
& +\beta_{20} \cdot V V_{j}+\beta_{21} \cdot V V_{j} \cdot \bar{a}_{i j}+\beta_{22} \cdot d d_{j} \cdot V V_{j} \\
& +\beta_{23} \cdot \operatorname{sex}_{j} \cdot V V_{j}+\beta_{24} \cdot S E S_{j} \cdot V V_{j}+\varepsilon_{i j}
\end{aligned}
$$

Here, $Y_{i j}$ represents cognitive performance on the Digit-Letter task of participant $j$ at age $i ; I, L S$, and $Q S$ are the intercept, linear slope, and quadratic slope, respectively; $\bar{a}_{i j}$ is each individual's age centered on all participants' mean age (84.92 years) and $\bar{a}_{i j}^{2}$ is the residualized component of each individual's age, squared, controlling for $\bar{a}_{i j}^{2} \bar{a}_{i j} ; \beta_{I}$ to $\beta_{I O}$ are the coefficients associated to the 10

${ }^{1}$ This indicator had values $0,1, \ldots, 10$ at occasions $1,2, \ldots, 11$. 
retest dummy variables $r_{1}$ to $r_{10} ; \beta_{11}, \beta_{12}$, and $\beta_{13}$ estimate the effects due to probable dementia diagnosis $(d d)$, sex, and SES (ses), respectively; $\beta_{14}, \beta_{15}$, and $\beta_{16}$ estimate the interactions between these covariates and mean age, and $\beta_{17}, \beta_{18}$ and $\beta_{19}$ estimate the interactions between these covariates; $\beta_{20}$ and $\beta_{21}$ estimate the effect of being homozygous for the Val allele $(V V)$ and its interaction with mean age relative to being a Met carrier; $\beta_{22}, \beta_{23}$, and $\beta_{24}$ estimate the interactions of $d d$, sex, and ses with $V V$; and finally, $\varepsilon_{i j}$ represents the error term.

We tested for random effects for the intercept $(I)$, linear age $(L S)$, and age squared ( $Q S$; i.e., variance in the intercept, linear, and quadratic slopes) and found the last not to be significant. We thus only tested predictors of individual differences in the intercept and in linear change (the latter predictors are henceforth referred to as interactions with age).

We used the full information maximum likelihood (FIML) algorithm to handle incomplete data (Arbuckle, 1996; Finkbeiner, 1979). This algorithm is standard practice in random effects models and allows estimating all parameters without imputing any data or excluding any participant. The estimation reduces bias to the extent that covariates related to data incompleteness are included in the model (Enders, 2001; Graham, 2009). The covariates considered here have been shown to be good predictors of attrition in the BASE (Ghisletta, McArdle, \& Lindenberger, 2006; Lindenberger et al., 2002).

\section{Results}

The 362 participants provided a maximum of 1,720 observations for the task. Table 2 shows the parameter estimates of the linear mixed effects model on Digit-Letter. As expected, both linear and quadratic fixed effects of age were significant and negative, indicating an average curvilinear decline trajectory, which was attenuated by positive retest effects up to the ninth assessment. The last two retest effects were negative, indicating that they were mimicking age effects. Note that the age heterogeneity of the sample is reduced at later measurement occasions, and that participants' age correlates highly with their time in the study (cf. Table 1), rendering the independent assessment of retest effects increasingly difficult. We thus excluded the last two retest dummy codes from the model.

Controlling for all other covariates, being likely affected by dementia implies a strong decrease of almost 18 points (nearly 2 $S D)$ compared with nondiagnosed participants. There were no significant sex or socioeconomic effects on overall performance.

Table 2

Results of the Linear Mixed-Effects Model for Digit-Letter

\begin{tabular}{|c|c|c|c|c|c|c|}
\hline & & Parm. est. & $S E$ & $d f$ & $t$ & $p$ \\
\hline \multicolumn{7}{|l|}{ Fixed effects } \\
\hline Intercept & $\left(I_{j}\right)$ & 42.99 & 5.06 & 329 & 8.49 & $<.0001$ \\
\hline \multirow[t]{2}{*}{ Age } & $\bar{a}_{i j}\left(L S_{j}\right)$ & -1.06 & 0.29 & 1366 & -3.66 & 0.0003 \\
\hline & $\bar{a}_{i j}^{2}\left(Q S_{j}\right)$ & -0.04 & 0.01 & 1366 & -7.17 & $<.0001$ \\
\hline \multirow[t]{8}{*}{ Retest effects } & $r_{l i}\left(\beta_{1}\right)$ & 0.89 & 0.24 & 1366 & 3.72 & 0.0002 \\
\hline & $r_{2 i}\left(\beta_{2}\right)$ & 0.58 & 0.27 & 1366 & 2.14 & 0.0325 \\
\hline & $r_{3 i}\left(\beta_{3}\right)$ & 0.91 & 0.31 & 1366 & 2.93 & 0.0035 \\
\hline & $r_{4 i}\left(\beta_{4}\right)$ & 1.38 & 0.33 & 1366 & 4.18 & $<.0001$ \\
\hline & $r_{5 i}\left(\beta_{5}\right)$ & 1.29 & 0.36 & 1366 & 3.55 & 0.0004 \\
\hline & $r_{6 i}\left(\beta_{6}\right)$ & 1.91 & 0.39 & 1366 & 4.91 & $<.0001$ \\
\hline & $r_{7 i}\left(\beta_{7}\right)$ & 1.76 & 0.48 & 1366 & 3.68 & 0.0002 \\
\hline & $r_{8 i}\left(\beta_{8}\right)$ & 2.29 & 0.50 & 1366 & 4.60 & $<.0001$ \\
\hline \multirow[t]{3}{*}{ Covariates } & $d d_{j}\left(\beta_{11}\right)$ & -17.91 & 5.66 & 329 & -3.16 & 0.0017 \\
\hline & $\operatorname{sex}_{j}\left(\beta_{12}\right)$ & -0.31 & 5.30 & 329 & -0.06 & 0.9540 \\
\hline & $S E S_{j}\left(\beta_{13}\right)$ & 0.13 & 0.09 & 329 & 1.43 & 0.1524 \\
\hline \multirow[t]{3}{*}{ Age interactions } & $d d_{j} \cdot \bar{a}_{i j}\left(\beta_{I 4}\right)$ & -0.08 & 0.11 & 1366 & -0.75 & 0.4547 \\
\hline & $\operatorname{sex}_{j} \cdot \bar{a}_{i j}\left(\beta_{15}\right)$ & 0.02 & 0.10 & 1366 & 0.22 & 0.8234 \\
\hline & $S E S_{j} \cdot \bar{a}_{i j}\left(\beta_{16}\right)$ & $>0.01$ & $>0.01$ & 1366 & 0.36 & 0.7154 \\
\hline \multirow[t]{3}{*}{ Cov. interactions } & $d d_{j} \cdot \operatorname{sex}_{j}\left(\beta_{17}\right)$ & -2.17 & 2.15 & 329 & -1.01 & 0.3133 \\
\hline & $d d_{j} \cdot S E S_{j}\left(\beta_{18}\right)$ & 0.26 & 0.10 & 329 & 2.50 & 0.0131 \\
\hline & $\operatorname{sex}_{j} \cdot S E S_{j}\left(\beta_{19}\right)$ & 0.04 & 0.10 & 329 & 0.39 & 0.6945 \\
\hline \multirow[t]{5}{*}{ Genetic influences } & $V V_{j}\left(\beta_{2 o}\right)$ & -0.07 & 5.30 & 329 & -0.01 & 0.9898 \\
\hline & $V V_{j} \cdot \bar{a}_{j}\left(\beta_{21}\right)$ & 0.23 & 0.10 & 1366 & 2.22 & 0.0269 \\
\hline & $d d_{j} \cdot V V_{j}\left(\beta_{22}\right)$ & 2.06 & 2.17 & 329 & 0.95 & 0.3423 \\
\hline & $\operatorname{sex}_{j} \cdot V V_{j}\left(\beta_{23}\right)$ & 1.39 & 1.97 & 329 & 0.70 & 0.4815 \\
\hline & $S E S_{j} \cdot V V_{j}\left(\beta_{24}\right)$ & -0.01 & 0.09 & 329 & -0.08 & 0.9338 \\
\hline Random effects & & Parm. est. & $S E$ & & $z$ & $p$ \\
\hline \multirow[t]{3}{*}{ Level 2} & Intercept & 59.91 & 5.76 & & 10.20 & $<.0001$ \\
\hline & age $\left(\bar{a}_{i j}\right)$ & .36 & .06 & & 6.5 & $<.0001$ \\
\hline & $\sigma\left(\right.$ Intercept, $\left.\bar{a}_{i j}\right)$ & $1.45(r=.31)$ & .44 & & 3.31 & .0009 \\
\hline Level 1 & residual $\left(\varepsilon_{i j}\right)$ & 9.69 & .43 & & 22.68 & $<.0001$ \\
\hline
\end{tabular}

Note. The predictors refer to the equation of the LMEM described in the text. Sex was coded $0=$ males, $1=$ females. VV was coded Val $/$ Val homozygotes $=1$, Met carriers $=0$. Parm. est. $=$ parameter estimate; $S E=$ standard error; $d f=$ degrees of freedom; $t=$ value of Student's t statistic; $p=p$ value corresponding to the null hypothesis that $\beta_{k}=0$ for the $k$ th predictor; cov. $=$ covariates; $\sigma=$ covariance; $r=$ correlation; $\mathrm{dd}=$ positive probable dementia diagnosis. 
However, dementia diagnosis interacted with SES, such that for diagnosed individuals, higher status was associated with better performance than lower status.

Counter to expectations, the Val/Met polymorphism did not influence the intercept term of speed performance. Accordingly, at the first assessment the cross-sectional scores did not differ reliably by genotype, though the sign of the difference was in the expected direction ( $t=-1.01, p=.32$, Cohen's $d=.12$ ). However, in line with our predictions, Val homozygotes declined less in speed performance than Met carriers (.83 vs. 1.06 per year). This effect was not moderated by other covariates. In terms of effect size, the $\mathrm{Val} / \mathrm{Val}$ versus any Met difference predicted $2.20 \%$ of the variance in speed decline.

To check the robustness of the results and their potential interaction with dementia status, we also performed the analyses on the subsample of $235(65 \%)$ participants who were never classified as likely demented (hence, for whom $d d_{j}=0$ ). The Val/Val versus any Met contrast continued to predict individual differences in change $(\beta=.24, p=.0373)$, and the effect size increased from $2.20 \%$ to $3.41 \%$. The remaining effects were virtually unchanged, except for consistently higher retest effects, as can be expected for individuals not diagnosed with probable dementia.

Figure 1 shows the individual trajectories of cognitive performance by age and the expected sample trajectories for negative (two upper lines) versus positive dementia diagnosis (two lower lines), and for $\mathrm{Val} / \mathrm{Val}$ homozygous individuals (continuous lines) versus Met carriers (dashed lines). The dementia diagnosis effect is pronounced throughout the whole age span considered, while the $\mathrm{Val} / \mathrm{Val}$ advantage is especially visible in advanced old age (over 85 years).

\section{Discussion}

In this study, we used a candidate gene approach to assess the association between the common Val66Met polymorphism of the

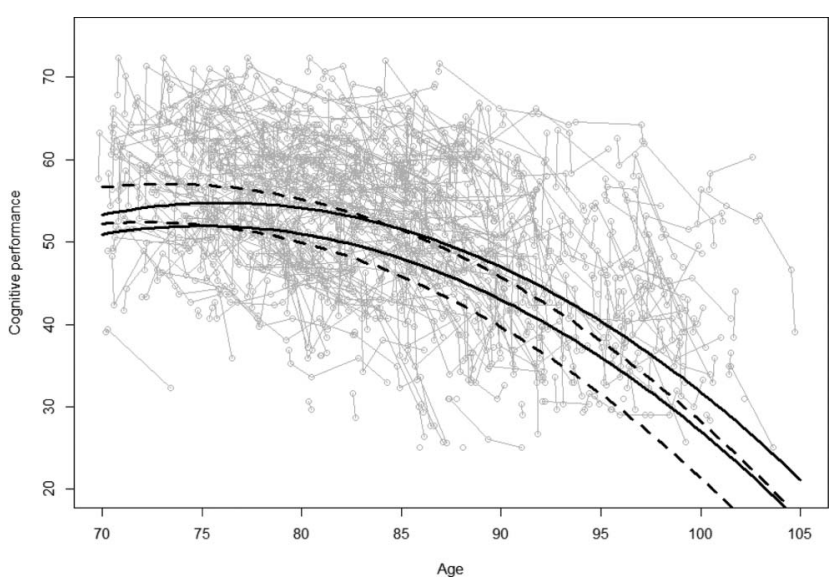

Figure 1. Digit-letter performance by age. Gray dots are individual measurements. Gray lines join data belonging to the same individual. Continuous black lines represent expected sample trajectories for Val homozygous individuals, while dashed lines are for Met carriers. The two upper lines represent expected sample trajectories for individuals not diagnosed with dementia, whereas the two lower lines are for diagnosed individuals.
BDNF gene and cognitive decline in a sample of 376 old and very old adults assessed up to 11 times over 13 years. In the BASE subsample for which genetic data were available, Met carriers evinced lower performance at Time 1, compared with Val homozygotes, but the difference was not statistically significant. This lack of result may be due to the satisfactory, though not very large, sample size of the study, or to the separation within the linear mixed effects modeling approach of level and change estimates. Nevertheless, being a Met carrier was associated with a steeper longitudinal rate of decline in perceptual speed compared with Val homozygotes. This effect, albeit weak $(2.20 \%$ of the variance in change was accounted for by this SNP alone), was robust, because it replicated in the subsample of individuals never classified as likely demented (there the effect increased to $3.41 \%$ of the variance in change). As shown in Figure 1, the deleterious genetic effect appears mainly in advanced old age, after 85 years (where the continuous black lines for Val homozygotes are superior to the dashed lines for Met carriers).

Given the particular sensitivity of the Digit-Letter task to detrimental age effects, we expected a reliable gene effect in favor of Val homozygotes in this very old sample. Unfortunately, there were no volumetric data to examine physiological correlates of genetic variation. It would have been interesting to know whether particular cerebral regions, especially the hippocampus, were, on the average, smaller in volume in Met carriers than in Val homozygotes. Lim et al. (2013) found this to be the case in adult Met carriers with high beta-amyloid who also had lower cognitive performance than Val homozygotes. Thus, our results are in agreement with those of Lim et al.

Our major finding is in accordance with the resource modulation hypothesis advanced by Lindenberger et al. (2008), which states that the effect of genetic variation on cognitive performance may be amplified in late adulthood when neurochemical, anatomical, and functional brain resources are declining and individual differences in performance are increasing. Hence, age-related loss of neurochemical and anatomical brain resources may amplify the genetic effects of polymorphisms on cognition. The results of Sambataro et al. (2010), who found that age-related changes in hippocampal function were greater for BDNF rs6265 Met carriers than for Val homozygotes, are also consistent with this hypothesis. Thus far, the resource modulation of BDNF effects has almost exclusively been tested in between-person, cross-sectional designs (e.g., Li et al., 2010). This study extends and investigates this phenomenon in a within-person, longitudinal framework. Our main result replicates and extends results from a number of between-person cross-sectional studies showing that Met allele carriers have lower episodic memory performance (Dempster et al., 2005; Echeverria et al., 2005; Egan et al., 2003; Hariri et al., 2003; Li et al., 2010; Miyajima et al., 2008; Tan et al., 2005), delayed recall (Miyajima et al., 2008), working memory (Echeverria et al., 2005; Rybakowski et al., 2003; Rybakowski et al., 2006), general intelligence (Tsai et al., 2004), and perceptual speed (Miyajima et al., 2008; Raz et al., 2009) compared with Val/Val homozygotes. In contrast to this earlier work, the present study examined genetic effects on individual differences in withinperson cognitive decline (by analyzing how individuals change across time as they age, rather than analyzing how individuals of different ages are different at a given time point). Future work needs to test the generality of the present findings by examining 
genetic effects on individual differences in growth and decline of other cognitive abilities, and at other ages such as childhood, adolescence, and earlier periods of adulthood, to test more thoroughly predictions of the resource modulation hypothesis.

We found no Sex $\times$ SNP interaction in our analysis, or interactions of the genetic association with SES or with having a likely dementia diagnosis. Albeit the robustness of our result, one may wonder about the relative small size of the actual effect. The present effect size is, however, typical of genetic association results. Indeed, rarely do such associations account for more than $2 \%$ or $3 \%$ of the variance in the trait (Plomin, DeFries, et al., 2013). As pointed out by several authors, these low effect sizes stand in clear contrast to those of heritability estimates from twin and adoption studies, which, for general cognitive abilities, are around 50\% (e.g., Harris \& Deary, 2011; Hunt, 2011; Payton, 2009; Plomin, DeFries, et al., 2013; Turkheimer, 2011; Turkheimer et al., 2003). The two sets of contrasting effect sizes are at the heart of the missing-heritability problem (Maher, 2008; Turkheimer, 2011). In sum, despite very large heritability estimates from twin and adoption studies, the genes supposedly driving such estimates have, by far, not been determined (even though approximately 20,000 to 25,000 human genes have been identified within the Human Genome Project). A likely source of disagreement may reside in the typical assumptions of twin and adoption studies. For instance, these studies usually assume no Gene $\times$ Environment interactions, whereas in reality, such interactions are likely to exist (Freund et al., 2013). Nevertheless, environmental variables that may be associated with cognitive performance are typically not measured in quantitative behavior studies, which thus cannot assess the viability of the "no Gene $\times$ Environment interaction" assumption (Hunt, 2011).

Recently, Visscher, Yang, and collaborators (Visscher, Yang, \& Goddard, 2010; Yang et al., 2010; Yang, Lee, Goddard, \& Visscher, 2011) proposed an alternative method, implemented in their software called genomewide complex-trait analysis (GCTA), which aims at predicting the similarity in a given trait for each pair of unrelated individuals, typically sharing less than $1 \%$ of genetic similarity, in a (very large) sample based on the total SNP similarity between each pair. This method allows obtaining heritability estimates that are typically larger than those from molecular approaches, and closer to those from behavior genetic studies. Initial estimates of heritability of general cognitive ability from GCTA vary from .24 to .48 , hence much closer to the .50 estimate from twin and adoption studies than the extremely low effects from GWAS (Deary et al., 2012; Petrill, 2002; Plomin, Haworth, et al., 2013). Nevertheless, if GCTA and twin-based estimates are directly compared, the former are constantly lower than the latter (by $26 \%$ to $56 \%$ in Plomin, Haworth, et al., 2013) and are estimated with much less precision (heritability estimates have much wider standard errors in GCTA than in twin-based studies).

A recent review of genetic effects on normal cognitive aging by Payton (2009) concluded that while most extant studies focus on single polymorphisms in cognition, future studies ought to seriously consider epistasis/gene-environment interactions to further our knowledge and reduce the missing-heritability problem. Several environmental factors may also influence the potential of expression of a trait, thereby influencing the phenotype expected for a given genotype. Hence, the same genotype may express differently in different, or even identical, environments (Freund et al., 2013; Hunt, 2011; Plomin, Haworth, et al., 2013). Moreover, it is recognized that for continuous traits, such as cognitive abilities, there is potential for additive genetic effects. Clearly, traits as complex as cognition are influenced by several dozens, if not thousands, of genes, rather than by a single gene. It is thus realistic to assume that multiple genes affect the same phenotypical trait.

In conclusion, we present, evidence in favor of an association between common Val66Met missense polymorphism (rs6265) of the BDNF gene and decline in cognitive performance in a longitudinal sample of old and very old adults. The result extends the resource modulation hypothesis of genetic effects on cognition to the assessment of individual differences in change, and awaits replication in studies with larger samples with both lower longitudinal dropout and younger participants.

\section{References}

Arbuckle, J. L. (1996). Full information estimation in the presence of incomplete data. In G. A. Marcoulides \& R. E. Schumacker (Eds.), Advanced structural equation modeling: Issues and techniques (pp. 243-277). Mahwah, NJ: Erlbaum.

Baltes, P. B., \& Mayer, K. U. (1999). The Berlin Aging Study: Aging from 70 to 100. New York, NY: Cambridge University Press.

Bryk, A. S., \& Raudenbush, S. W. (1987). Application of hierarchical linear models to assessing change. Psychological Bulletin, 101, 147158. doi:10.1037/0033-2909.101.1.147

Bueller, J. A., Aftab, M., Sen, S., Gomez-Hassan, D., Burmeister, M., \& Zubieta, J.-K. (2006). BDNF Val66Met allele is associated with reduced hippocampal volume in healthy subjects. Biological Psychiatry, 59, 812-815. doi:10.1016/j.biopsych.2005.09.022

Colzato, L. S., van den Wildenberg, W. P. M., \& Hommel, B. (2013). The genetic impact (C957T-DRD2) on inhibitory control is magnified by aging. Neuropsychologia, 51, 1377-1381.

Davies, G., Tenesa, A., Payton, A., Yang, J., Harris, S. E., Liewald, D., . . Deary, I. J. (2011). Genome-wide association studies establish that human intelligence is highly heritable and polygenic. Molecular Psychiatry, 16, 996-1005. doi:10.1038/mp.2011.85

Deary, I. J., Yang, J., Davies, G., Harris, S. E., Tenesa, A., Liewald, D., . . Visscher, P. M. (2012). Genetic contributions to stability and change in intelligence from childhood to old age. Nature, 482, 212-215.

Dempster, E., Toulopoulou, T., McDonald, C., Bramon, E., Walshe, M., Filbey, F., . . . Collier, D. A. (2005). Association between BDNF Val66Met genotype and episodic memory. American Journal of Medical Genetics. Part B, Neuropsychiatric Genetics, 134, 73-75. doi:10.1002/ ajmg.b. 30150

Echeverria, D., Woods, J. S., Heyer, N. J., Rohlman, D. S., Farin, F. M., Bittner, A. C., Jr., . . Garabedian, C. (2005). Chronic low-level mercury exposure, BDNF polymorphism, and associations with cognitive and motor function. Neurotoxicology and Teratology, 27, 781-796. doi: 10.1016/j.ntt.2005.08.001

Egan, M. F., Kojima, M., Callicott, J. H., Goldberg, T. E., Kolachana, B. S., Bertolino, A., .. . Weinberger, D. R. (2003). The BDNF Val66Met polymorphism affects activity-dependent secretion of BDNF and human memory and hippocampal function. Cell, 112, 257-269. doi:10.1016/ S0092-8674(03)00035-7

Enders, C. K. (2001). A primer on maximum likelihood algorithms available for use with missing data. Structural Equation Modeling, 8, 128141. doi:10.1207/S15328007SEM0801_7

Erickson, K. I., Kim, J. S., Suever, B. L., Voss, M. W., Francis, B. M., \& Kramer, A. F. (2008). Genetic contributions to age-related decline in executive function: A 10-year longitudinal study of COMT and BDNF polymorphisms. Frontiers in Human Neuroscience, 2, 11. doi:10.3389/ neuro.09.011.2008 
Fan, X. (2003). Power of latent growth modeling for detecting group differences in linear growth trajectory parameters. Structural Equation Modeling, 10, 380-400. doi:10.1207/S15328007SEM1003_3

Ferrer, E., Salthouse, T. A., McArdle, J. J., Stewart, W. F., \& Schwartz, B. S. (2005). Multivariate modeling of age and retest in longitudinal studies of cognitive abilities. Psychology and Aging, 20, 412-422. doi:10.1037/0882-7974.20.3.412

Finkbeiner, C. (1979). Estimation for the multiple factor model when data are missing. Psychometrika, 44, 409-420. doi:10.1007/BF02296204

Finkel, D., Reynolds, C. A., McArdle, J. J., Hamagami, F., \& Pedersen, N. L. (2009). Genetic variance in processing speed drives variation in aging of spatial and memory abilities. Developmental Psychology, 45, 820-834. doi:10.1037/a0015332

Freund, J., Brandmaier, A. M., Lewejohann, L., Kirste, I., Kritzler, M., Krüger, A., . . Kempermann, G. (2013). Emergence of individuality in genetically identical mice. Science, 340, 756-759. doi:10.1126/science .1235294

Frodl, T., Schüle, C., Schmitt, G., Born, C., Baghai, T., Zill, P., . . . Meisenzahl, E. M. (2007). Association of the brain-derived neurotrophic factor Val66Met polymorphism with reduced hippocampal volumes in major depression. Archives of General Psychiatry, 64, 410-416. doi: 10.1001/archpsyc.64.4.410

Gerstorf, D., Herlitz, A., \& Smith, J. (2006). Stability of cognitive sex differences in advanced old age: The role of education and attrition. Journal of Gerontology: Psychological Sciences, 61, P245-P249. doi: 10.1093/geronb/61.4.P245

Ghisletta, P., McArdle, J. J., \& Lindenberger, U. (2006). Longitudinal cognition-survival relations in old and very old age. European Psychologist, 11, 204-223. doi:10.1027/1016-9040.11.3.204

Ghisletta, P., Rabbitt, P., Lunn, M., \& Lindenberger, U. (2012). Two thirds of the age-based changes in fluid and crystallized intelligence, perceptual speed, and memory in adulthood are shared. Intelligence, 40, 260268. doi:10.1016/j.intell.2012.02.008

Graham, J. W. (2009). Missing data analysis: Making it work in the real world. Annual Review of Psychology, 60, 549-576. doi:10.1146/annurev .psych.58.110405.085530

Hariri, A. R., Goldberg, T. E., Mattay, V. S., Kolachana, B. S., Callicott, J. H., Egan, M. F., \& Weinberger, D. R. (2003). Brain-derived neurotrophic factor Val66Met polymorphism affects human memory-related hippocampal activity and predicts memory performance. The Journal of Neuroscience: The Official Journal of the Society for Neuroscience, 23, 6690-6694.

Harris, S. E., \& Deary, I. J. (2011). The genetics of cognitive ability and cognitive ageing in healthy older people. Trends in Cognitive Sciences, 15, 388-394.

Helmchen, H., Baltes, M. M., Geiselmann, B., Kanowski, S., Linden, M., \& Reischies, F. M. (1999). Psychiatric illnesses in old age. In P. B. Baltes \& K. U. Mayer (Eds.), The Berlin Aging Study: Aging from 700 to 100 (pp. 167-196). New York, NY: Cambridge University Press.

Heston, L. L. (1966). Psychiatric disorders in foster home reared children of schizophrenic mothers. The British Journal of Psychiatry, 112, 819825. doi:10.1192/bjp.112.489.819

Holland, P. M., Abramson, R. D., Watson, R., \& Gelfand, D. H. (1991). Detection of specific polymerase chain reaction product by utilizing the $5^{\prime}-3^{\prime}$ exonuclease activity of Thermus aquaticus DNA polymerase. PNAS Proceedings of the National Academy of Sciences of the United States of America, 88, 7276-7280. doi:10.1073/pnas.88.16.7276

Hultsch, D. F. (2004). Introduction to special issue on longitudinal studies of cognitive aging. Aging, Neuropsychology, and Cognition, 11, 101103. doi: $10.1080 / 13825580490510973$

Hunt, E. B. (2011). Human intelligence. Cambridge, UK: Cambridge University Press.

Laird, N. M., \& Ware, J. H. (1982). Random-effects models for longitudinal data. Biometrics, 38, 963-974. doi:10.2307/2529876
Li, S.-C., Chicherio, C., Nyberg, L., von Oertzen, T., Nagel, I. E., Papenberg, G., . . . Bäckman, L. (2010). Ebbinghaus revisited: Influences of the BDNF Val66Met polymorphism on backward serial recall are modulated by human aging. Journal of Cognitive Neuroscience, 22, 2164 2173. doi:10.1162/jocn.2009.21374

Li, S.-C., Papenberg, G., Nagel, I. E., Preuschhof, C., Schröder, J., Nietfeld, W., . . . Bäckman, L. (2013). Aging magnifies the effects of dopamine transporter and D2 receptor genes on backward serial memory. Neurobiology of Aging, 34, 358, e1-10.

Lim, Y. Y., Villemagne, V. L., Laws, S. M., Ames, D., Pietrzak, R. H., Ellis, K. A., . . Maruff, P. (2013). BDNF Val66Met, Aß amyloid, and cognitive decline in preclinical Alzheimer's disease. Neurobiology of Aging, 34, 2457-2464. doi:10.1016/j.neurobiolaging.2013.05.006

Lindenberger, U., \& Ghisletta, P. (2009). Cognitive and sensory declines in old age: Gauging the evidence for a common cause. Psychology and Aging, 24, 1-16. doi:10.1037/a0014986

Lindenberger, U., Mayr, U., \& Kliegl, R. (1993). Speed and intelligence in old age. Psychology and Aging, 8, 207-220. doi:10.1037/0882-7974.8 .2 .207

Lindenberger, U., Nagel, I. E., Chicherio, C., Li, S.-C., Heekeren, H. R., \& Bäckman, L. (2008). Age-related functioning in brain resources modulates genetic effects on cognitive functioning. Frontiers in Neuroscience, 2, 234-244. doi:10.3389/neuro.01.039.2008

Lindenberger, U., Singer, T., \& Baltes, P. B. (2002). Longitudinal selectivity in aging populations: Separating mortality-associated versus experimental components in the Berlin Aging Study (BASE). The Journals of Gerontology: Series B: Psychological Sciences and Social Sciences, 57, P474-P482. doi:10.1093/geronb/57.6.P474

Lindenberger, U., Smith, J., Mayer, K. U., \& Baltes, P. B. (2010). Die Berliner Alterstudie [The Berlin Aging Study]. Berlin, Germany: Akademie Verlag.

Lövdén, M., Ghisletta, P., \& Lindenberger, U. (2004). Cognition in the Berlin Aging Study (BASE): The first 10 years. Aging, Neuropsychology, and Cognition, 11, 104-133. doi:10.1080/13825580490510982

Lövdén, M., Schaefer, S., Noack, H., Kanowski, M., Kaufmann, J., Tempelmann, C., . . . Bäckman, L. (2011). Performance-related increases in hippocampal $\mathrm{N}$-acetylaspartate (NAA) induced by spatial navigation training are restricted to BDNF Val homozygotes. Cerebral Cortex, 21 , 1435-1442. doi:10.1093/cercor/bhq230

Maher, B. (2008). Personal genomes: The case of the missing heritability. Nature, 456, 18-21.

McArdle, J. J., Prescott, C. A., Hamagami, F., \& Horn, J. L. (1998). A contemporary method for developmental-genetic analyses of age changes in intellectual abilities. Developmental Neuropsychology, 14, 69-114. doi:10.1080/87565649809540701

Miyajima, F., Ollier, W., Mayes, A., Jackson, A., Thacker, N., Rabbitt, P., . . . Payton, A. (2008). Brain-derived neurotrophic factor polymorphism Val66Met influences cognitive abilities in the elderly. Genes, Brain, and Behavior, 7, 411-417. doi:10.1111/j.1601-183X.2007.00363.x

Neiderhiser, J. M. (2001). Understanding the roles of genome and envirome: Methods in genetic epidemiology. The British Journal of Psychiatry. Supplement, 178, s12-s17. doi:10.1192/bjp.178.40.s12

Papenberg, G., Bäckman, L., Nagel, I. E., Nietfeld, W., Schröder, J., Bertram, L., . . . Li, S.-C. (2013). Dopaminergic gene polymorphisms affect long-term forgetting in old age: Further support for the magnification hypothesis. Journal of Cognitive Neuroscience, 25, 571-579. doi:10.1162/jocn_a_00359

Payton, A. (2009). The impact of genetic research on our understanding of normal cognitive ageing: 1995 to 2009. Neuropsychology Review, 19, 451-477. doi:10.1007/s11065-009-9116-Z

Petrill, S. A. (2002). The case for general intelligence: A behavioral genetic perspective. In R. J. Sternberg \& E. L. Grigorenko (Eds.), The general factor of intelligence (pp. 281-298). Mahwah, NJ: Erlbaum. 
Pezawas, L., Verchinski, B. A., Mattay, V. S., Callicott, J. H., Kolachana, B. S., Straub, R. E., . . . Weinberger, D. R. (2004). The brain-derived neurotrophic factor Val66Met polymorphism and variation in human cortical morphology. The Journal of Neuroscience: The Official Journal of the Society for Neuroscience, 24, 10099-10102. doi:10.1523/ JNEUROSCI.2680-04.2004

Plomin, R., DeFries, J. C., Knopik, V. S., \& Neiderhiser, J. M. (2013). Behavioral genetics (6th ed.). New York, NY: Worth.

Plomin, R., Haworth, C. M. A., Meaburn, E. L., Price, T. S., Wellcome Trust Case Control Consortium 2, \& Davis, O. S. P. (2013). Common DNA markers can account for more than half of the genetic influence on cognitive abilities. Psychological Science, 24, 562-568. doi:10.1177/ 0956797612457952

Raz, N., Rodrigue, K. M., Kennedy, K. M., \& Land, S. (2009). Genetic and vascular modifiers of age-sensitive cognitive skills: Effects of COMT, BDNF, ApoE, and hypertension. Neuropsychology, 23, 105-116. doi: 10.1037/a0013487

Rybakowski, J. K., Borkowska, A., Czerski, P. M., Skibińska, M., \& Hauser, J. (2003). Polymorphism of the brain-derived neurotrophic factor gene and performance on a cognitive prefrontal test in bipolar patients. Bipolar Disorders, 5, 468-472. doi:10.1046/j.1399-5618.2003 .00071.x

Rybakowski, J. K., Borkowska, A., Skibińska, M., Szczepankiewicz, A., Kapelski, P., Leszczynska-Rodziewicz, A., . . . Hauser, J. (2006). Prefrontal cognition in schizophrenia and bipolar illness in relation to Val66Met polymorphism of the brain-derived neurotrophic factor gene. Psychiatry and Clinical Neurosciences, 60, 70-76. doi:10.1111/j.14401819.2006.01462.x

Salthouse, T. A. (1996). The processing-speed theory of adult age differences in cognition. Psychological Review, 103, 403-428. doi:10.1037/ 0033-295X.103.3.403

Sambataro, F., Murty, V. P., Lemaitre, H. S., Reed, J. D., Das, S., Goldberg, T. E., . . Mattay, V. S. (2010). BNDF modulates normal human hippocampal ageing. Molecular Psychiatry, 15, 116-118. doi: 10.1038/mp.2009.64

Schaie, K. W., \& Hofer, S. M. (2001). Longitudinal studies in aging research. In J. E. Birren \& K. W. Schaie (Eds.), Handbook of the psychology of aging (5th ed., pp. 53-77). San Diego, CA: Academic Press.

Snijders, T. A. B., \& Bosker, R. J. (1999). Multilevel analysis: An introduction to basic and advanced multilevel modeling. London, UK: Sage.

Tan, Y. L., Zhou, D. F., Cao, L. Y., Zou, Y. Z., Wu, G. Y., \& Zhang, X. Y. (2005). Effect of the BDNF Val66Met genotype on episodic memory in schizophrenia. Schizophrenia Research, 77, 355-356. doi:10.1016/j schres.2005.03.012

Tsai, S.-J., Hong, C.-J., Yu, Y. W.-Y., \& Chen, T.-J. (2004). Association study of a brain-derived neurotrophic factor (BDNF) Val66Met polymorphism and personality trait and intelligence in healthy young females. Neuropsychobiology, 49, 13-16. doi:10.1159/000075333

Turkheimer, E. (2011). Still missing. Research in Human Development, 8 , 227-241. doi:10.1080/15427609.2011.625321

Turkheimer, E., Haley, A., Waldron, M., D'Onofrio, B., \& Gottesman, I. I. (2003). Socioeconomic status modifies heritability of IQ in young children. Psychological Science, 14, 623-628. doi:10.1046/j.0956-7976 2003.psci_1475.x

Visscher, P. M., Yang, J., \& Goddard, M. E. (2010). A commentary on "Common SNPs explain a large proportion of the heritability for human height" by Yang et al. (2010). Twin Research and Human Genetics, 13, 517-524. doi:10.1375/twin.13.6.517

Wechsler, D. (1955). Manual for the Wechsler Adult Intelligence Scale. New York, NY: The Psychological Corporation.

Yang, J., Benyamin, B., McEvoy, B. P., Gordon, S., Henders, A. K., Nyholt, D. R., . . Visscher, P. M. (2010). Common SNPs explain a large proportion of the heritability for human height. Nature Genetics, 42, 565-569. doi:10.1038/ng.608

Yang, J., Lee, S. H., Goddard, M. E., \& Visscher, P. M. (2011). GCTA: A tool for genome-wide complex trait analysis. American Journal of $\mathrm{Hu}$ man Genetics, 88, 76-82. doi:10.1016/j.ajhg.2010.11.011

Received May 30, 2013 Revision received September 16, 2013 Accepted October 17, 2013 